\title{
Contour reconstruction of three-dimensional spiral CT damage image
}

\author{
Cui Ling-Ling* and Zhang Hui \\ The First Affiliated Hospital of Jinzhou Medical University \\ Jinzhou 121001, P. R. China \\ *cuiawenn@163.com
}

Received 30 March 2018

Accepted 5 July 2018

Published 14 August 2018

\begin{abstract}
In order to improve the diagnosis and analysis ability of 3D spiral CT and to reconstruct the contour of 3D spiral CT damage image, a contour reconstruction method based on sharpening template enhancement for 3D spiral CT damage image is proposed. This method uses the active contour LasSO model to extract the contour feature of the 3D spiral CT damage image and enhances the information by sharpening the template enhancement technique and makes the noise separation of the 3D spiral CT damage image. The spiral CT image was processed with ENT, and the statistical shape model of 3D spiral CT damage image was established. The gradient algorithm is used to decompose the feature to realize the analysis and reconstruction of the contour feature of the 3D spiral CT damage image, so as to improve the adaptive feature matching ability and the ability to locate the abnormal feature points. The simulation results show that in the 3D spiral CT damage image contour reconstruction, the proposed method performs well in the feature matching of the output pixels, shortens the contour reconstruction time by $20 / \mathrm{ms}$, and provides a strong ability to express the image information. The normalized reconstruction error of CES is 30\%, which improves the recognition ability of $3 \mathrm{D}$ spiral CT damage image, and increases the signal-to-noise ratio of peak output by $40 \mathrm{~dB}$ over other methods.
\end{abstract}

Keywords: Spiral CT; three-dimensional image; contour feature extraction; sharpening template enhancement.

\section{Introduction}

With the development of medical image processing technology, medical image analysis is performed through image information processing and analysis methods to improve the diagnosis and analysis ability of medical pathology. Common medical images include X-ray images, CT images, spiral CT images and so on. Spiral CT is a product of the development of computer information processing technology. Multi-slices spiral CT imaging is adopted to perform digital projection of human organs to create projection data, so as to achieve

*Corresponding author.

This is an Open Access article published by World Scientific Publishing Company. It is distributed under the terms of the Creative Commons Attribution 4.0 (CC-BY) License. Further distribution of this work is permitted, provided the original work is properly cited. 
CT angiography, cardiac assessment, cerebral perfusion imaging, etc. Spiral CT imaging technology plays an important role in the detection of multifunctional organs and etiological analysis to bodies. ${ }^{1}$ Spiral CT imaging also plays an important role in the assessment of human organ damage. ${ }^{2,3}$ Pathological analysis of spiral CT imaging is based on the reconstruction of three-dimensional spiral $\mathrm{CT}$ image. Information feature extraction and reconstruction techniques of image are adopted to realize adaptive reconstruction of spiral CT image, thus realizing high-resolution $\mathrm{CT}$ imaging and angiography. Research on contour reconstruction of threedimensional spiral CT damage image has an important role in the treatment of injuries.

Currently, contour reconstruction methods of three-dimensional spiral CT damage image mainly include RGB image reconstruction method, block radon scale reconstruction method, point distribution model reconstruction method, similarity feature decomposition reconstruction method and so on. ${ }^{4}$ Based on the extraction of edge contour feature quantity of three-dimensional spiral CT damage image, three-dimensional reconstruction is performed to the spiral CT image through template feature matching and adaptive continuous scale transformation methods, to improve the recognition and statistical analysis ability of the image. In Ref. 5, a three-dimensional reconstruction method of spiral CT image based on dynamic feature analysis of dimensional coordinate points is proposed, which reconstructs detailed texture features of image and performs three-dimensional contour construction to the $\mathrm{CT}$ damage image to improve the feature recognition ability of the image. However, this method is computationally expensive and has bad feature recognition ability in contour reconstruction of three-dimensional spiral CT damage image. In Ref. 6, a contour reconstruction technology of three-dimensional spiral CT damage image based on background difference continuous reconstruction and RGB quantitative decomposition is proposed, which adopts the grid segmentation method to perform template matching processing to CT image and to perform contour retrieval based on internal texture structure information of the CT image, thus improving the edge contour reconstruction ability of the image. However, this method has large computation load in contour reconstruction and provides low recognition accuracy of the reconstructed image. In Ref. 7, a threedimensional reconstruction method of spiral CT image based on triangular Bernstein-Bezier surface fitting is proposed. Based on trigonometric Bernstein-Bezier surface fitting of a generated single triangular plate, the quadric interpolation of normal vector of triangular plate trihedral vertex is adopted to recalculate the normal vector of trigonometric Bernstein-Bezier surface, so that the display effect of the fitted surface is continuous and smooth. At the same time, the reconstructed surface model is subjected to plane cutting, and the cross-section and cut surface of the sectioned model are pasted with textures to add image information. However, there are problems of relatively low resolution of $\mathrm{CT}$ imaging and radiography. In Ref. 8, study is carried out to the process that ultrasonic is adopted for detection of components under test to obtain a two-dimensional image, to extract two-dimensional contour information and finally to form a three-dimensional image. Specific data acquisition methods or algorithms are given for each process. All tests show that it has good effect and can basically meet the requirements of extracting two-dimensional information. However, there is a problem that the expression ability of image information is weak.

In order to solve the above problems, a contour reconstruction method of three-dimensional spiral CT damage image based on sharpening template enhancement technique is proposed in this paper. First, noise separation is carried out to threedimensional spiral CT damage image, and features of the three-dimensional spiral CT damage image contours are extracted with an active contour Lasso model; then the sharpening template enhancement technique is adopted for information enhancement processing to the spiral CT image to establish a statistical shape model for the three-dimensional spiral CT damage image and the gradient algorithm method is adopted for feature decomposition to realize extraction and reconstruction of the contour features of the three-dimensional spiral CT damage image, so as to improve the adaptive feature matching ability and abnormal feature points location ability of the image. Finally, a simulation experiment is performed, which demonstrates the superior performance of the method proposed in this paper in improving the contour reconstruction ability of three-dimensional spiral CT damage image. 


\section{Noise Separation of 3D Spiral CT Damage Image}

\subsection{Information enhancement processing of spiral CT image}

To reconstruct the contour of 3D spiral CT damage image, we first need to separate the noise from 3D spiral CT damage image.

In this paper, a contour reconstruction method of three-dimensional spiral CT damage image based on the sharpening template enhancement technique is proposed, and then the feature component of edge information of the spiral CT damage image is obtained as follows:

$$
\text { is _visible }\left(M_{m i}, C_{j}\right)= \begin{cases}1, & \text { if }\left\{\begin{array}{l}
j \neq i, \\
C_{j} \text { may call } M_{m i} .
\end{array}\right. \\
0, & \text { otherwise }\end{cases}
$$

In the formula, $M_{m_{i}}$ is used to reconstruct the smoothing operator for spiral CT damage three-dimensional image, and $C_{j}$ represents the feature variables in the edge information of the image. The feature component of continuous reconstruction of the point $\left(a, b_{m}\right)$ in the three-dimensional spiral CT damage image surface is

$$
L\left(a, b_{m}\right) s=\log \left(\frac{|\mathbf{V}|\left|\mathbf{V}_{m} \cap \mathbf{V}_{n}\right|}{\left|\mathbf{V}_{m}\right|\left|\mathbf{V}_{n}\right|}\right) .
$$

In the formula, $\mathbf{V}$ is a vector, $\mathbf{V}_{m}$ is the eigenvector of variable $m$ and $\mathbf{V}_{n}$ is the eigenvector of variable $n$. The curvilinear path fusion tracking method $^{9}$ is adopted to perform continuous reconstruction to extract texture features of the threedimensional spiral CT damage image, namely:

$$
\begin{aligned}
L\left(a, b_{m}\right)= & \sum_{\mathbf{V}_{m} \in P^{\text {res }}} \sum_{\mathbf{V}_{n} \in P^{\text {true }}} \frac{\left|\mathbf{V}_{m} \cap \mathbf{V}_{n}\right|}{|\mathbf{V}|} \\
& \times \log \left(\frac{|\mathbf{V}|\left|\mathbf{V}_{m} \cap \mathbf{V}_{n}\right|}{\left|\mathbf{V}_{m}\right|\left|\mathbf{V}_{n}\right|}\right) .
\end{aligned}
$$

The spiral CT image is conducted with information enhancement, from which the approximate solution of differential feature of the threedimensional spiral CT damage image is obtained:

$$
\overline{x_{T}}=\frac{1}{T} \sum_{i=1}^{T} x_{i}
$$

where $x_{1}, x_{2}, x_{3}, \ldots, x_{T}$ is the feature subsample of each sub-block and $T$ is the feature characteristic component of edge information of the image. A statistical shape model of the three-dimensional spiral CT damage image is constructed, and two sets of adjacent pixels are obtained:

$$
F=\tilde{p}(x, y)=p(x, y)\left(\frac{v(x)}{v(y)}\right)^{1 / 2},
$$

where

$$
p(x, y)=\frac{k(x, y)}{v(x)}, \quad v(x)=\sum_{y} k(x, y) .
$$

In the formula, $k(x, y)$ is a pixel characteristic function. The neighborhood search method ${ }^{10}$ is adopted for rapid feature point location and information enhancement processing to the spiral CT image, and it is obtained that

$$
\begin{aligned}
E_{\text {int }(v i)}= & \frac{1}{2}\left(\partial i|d-| v i-v i-\left.1\right|^{2}\right. \\
& \left.+\beta i|v i-1+2 v i+v i+1|^{2}\right),
\end{aligned}
$$

where

$$
d=\frac{1}{n} \sum_{i=0}^{n-1}|v i-1|
$$

In the formula, $v_{i}$ represents the information location coordinate, $\partial_{i}$ represents the longitudinal enhancement coefficient and $\beta_{i}$ represents the transverse enhancement coefficient. Based on the above processing, information enhancement is achieved for the three-dimensional spiral CT damage image, which improves the recognizability of the image.

\subsection{Noise decomposition of three-dimensional spiral $C T$ damage image}

The image noise separation can improve the pixel of the damaged image, and the quality of the spiral CT damage image is improved, which is beneficial to the optimization of the 3D image contour reconstruction of the spiral CT damage. For an original collected three-dimensional spiral CT damage image, the wavelet denoising method ${ }^{11}$ is adopted for noise separation. The system of linear equations of image wavelet denoising is:

$$
\left(\begin{array}{lll}
\mathbf{x} & \mathbf{y} & \mathbf{z} \\
\mathbf{o} & \mathbf{o} & \mathbf{o}
\end{array}\right)=\left(\begin{array}{cc}
(\mathbf{H}+\boldsymbol{\tau} \cdot \mathbf{I}) & \mathbf{P} \\
\mathbf{P}^{T} & \mathbf{O}
\end{array}\right)\left(\begin{array}{ccc}
\boldsymbol{\gamma} & \theta & \omega \\
\mathbf{a} & \mathbf{b} & \mathbf{c}
\end{array}\right),
$$


where $x, y, z$ expressed as three-dimensional coordinate value, $o$ expressed as pixel reconstruction group with unknown edge contour region, $H$ expressed as 3D profile-space point imaging efficiency, $I$ expressed as damaged image pixel, $P$ expressed as image center, $P^{T}$ expressed as radial distortion coefficient of edge profile feature, $\gamma$ expressed as pixel conductance oefficient, $\theta$ expressed as phase angle of image interpolation transform, $a$ expressed as the proportion factor of the decomposition of the damage image and the edge feature, $b$ expressed as reconstruction area of 3D image with spiral CT injury, $c$ expressed as order central moment definition. The Harris corner detection method $^{12}$ is used to smooth the three-dimensional spiral CT damage image on the reconstruction surface of the image. The smoothing function is:

$$
\begin{aligned}
D(x, y, \sigma) & =(G(x, y, k \sigma)-G(x, y, \sigma)) * I(x, y) \\
& =L(x, y, k \sigma)-L(x, y, \sigma),
\end{aligned}
$$

where

$$
L(x, y, \sigma)=G(x, y, \sigma) \otimes I(x, y),
$$

where $D$ expressed as damaged image sampling set after smoothing, $\sigma$ expressed as surface density of three-dimensional image of spiral CT damage, $k$ expressed as Pose parameter. $I(x, y)$ is the gray value of the three-dimensional spiral CT damage image in $(x, y) ; L(x, y, \sigma)$ is the Taubin smoothing operator ${ }^{13}$ and $G(x, y, \sigma)$ is the block matching feature coefficient of the three-dimensional damage image. The formula is

$$
G(x, y, \sigma)=\frac{1}{2 \pi \sigma^{2}} e^{-\frac{\left(x^{2}+y^{2}\right)}{2 \sigma^{2}}} .
$$

By judging the wrinkle information of the threedimensional spiral CT damage image, and performing hierarchical decomposition of the image noise, the decomposition coefficient is obtained as follows according to the Euclidean distance $E(d(x, y))$ between wrinkles:

$$
F_{d}-\frac{d}{d x} F_{d_{x}}-\frac{d}{d y} F_{d_{y}}=0
$$

where $F_{d}$ represents the edge scale of the spiral CT damage image. The noise distance of the gird surface of the image is $d(x, y)$. The number of noises of the damage image is $N_{l}$, which is described as

$$
N_{l}= \begin{cases}1 & l=0, L \\ {\left[2 \pi \cdot \frac{D}{2} \cdot \sin \eta / l_{\text {triangle }}\right]} & l=1, \ldots, L-1\end{cases}
$$

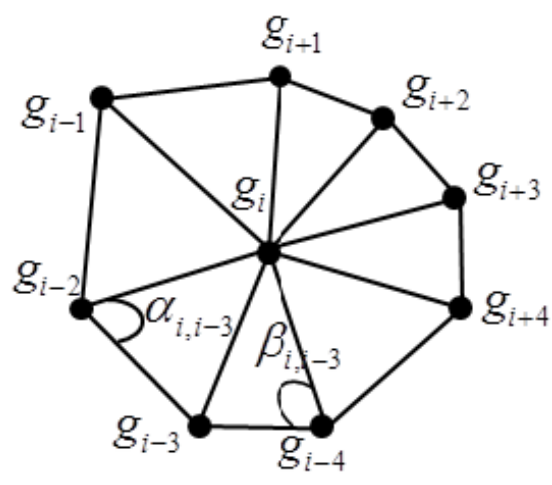

Fig. 1. Smooth filtering of three-dimensional spiral CT damage image.

Among them, $l$ is the calculation of the vertex distance of the mesh model and $l_{\text {triangle }}$ is its average value. According to the above analysis, the noise separation model shown in Fig. 1 is adopted to achieve smooth filtering of the three-dimensional spiral CT damage image.

The mathematical expression of the smooth filtered image output is as follows:

$$
\begin{aligned}
\mathbf{G}_{\text {new }} & =(1+\mu T)(1+\lambda T) \mathbf{G}_{\text {old }}, \\
T\left(g_{i}\right) & =\frac{1}{\sum_{k} \omega_{k}} \cdot \sum_{k} \omega_{k}\left(g_{k}-g_{i}\right),
\end{aligned}
$$

where $\mathbf{G}_{\text {new }}$ and $\mathbf{G}_{\text {old }}$ represent low-frequency and high-frequency components of the image, respectively, and $T\left(g_{i}\right)$ is the image degradation feature point and $\omega_{k}$ is the attenuation coefficient of the image.

\section{Optimization of Contour Reconstruction of Three-Dimensional Spiral CT Damage Image}

\subsection{Feature extraction of $3 D$ spiral $C T$ damage image contour}

Vector quantization feature quantity of the CT image is set, and gray pheromone of the image is extracted. ${ }^{14-16}$ Then the front $k$-dimensional feature of the three-dimensional spiral CT damage image is obtained and described as follows:

$$
P(\phi)=\int \frac{1}{2}(|\nabla \phi|-1)^{2} d x .
$$

In the formula, $\phi$ is the eigenvalue. With the sparse linear segmentation method, ${ }^{17}$ the reference template matching function for the image is obtained as 
follows:

$$
\operatorname{Data}(x, y, d(x, y))=|u(x-d(x, y), y)-\tilde{u}(x, y)|^{2},
$$

where $\tilde{u}$ represents the referenced template image; $u$ represents the image under reconstruction. Edge contour information of the CT image is reset and then the sparse group of linear equations is constructed for continuous segmentation as follows:

$$
\frac{\left\langle\tau_{d} u^{\prime}, \tilde{u}\right\rangle_{\varphi_{x_{0}}}}{\left\|\tau_{d} u\right\|_{\varphi_{x_{0}}}\|\tilde{u}\|_{\varphi_{x_{0}}}}-\frac{\left\langle\tau_{d} u, \tilde{u}\right\rangle_{\varphi_{x_{0}}}\left\langle\tau_{d} u^{\prime}, \tau_{d} u\right\rangle_{\varphi_{x_{0}}}}{\left\|\tau_{d} u\right\|_{\varphi_{x_{0}}}^{3}\|\tilde{u}\|_{\varphi_{x_{0}}}} .
$$

In the formula, $\tau$ is time-consuming for continuous segmentation, $u^{\prime}$ is the image after the reconfiguration. Thus, the sparse decomposition process of the three-dimensional spiral CT damage image is obtained as follows:

$$
\begin{aligned}
\frac{\partial}{\partial d}( & \left.\frac{\left\langle\tau_{d} u, \tilde{u}\right\rangle_{\varphi_{x_{0}}}}{\left\|\tau_{d} u\right\|_{\varphi_{x_{0}}}\|\tilde{u}\|_{\varphi_{x_{0}}}}\right) \\
= & \frac{\left(\left\|\tau_{d} u\right\|_{\varphi_{x_{0}}} \partial / \partial d\left(\left\langle\tau_{d} u, \tilde{u}\right\rangle_{\varphi_{x_{0}}}\right)\right.}{\left(\left\|\tau_{d} u\right\|_{\varphi_{x_{0}}}\right)^{2}\|\tilde{u}\|_{\varphi_{x_{0}}}} \\
= & \frac{\partial / \partial d\left(\left\langle\tau_{d} u, \tilde{u}\right\rangle_{\varphi_{x_{0}}}\right.}{\left\|\tau_{d} u\right\|_{\varphi_{x_{0}}}\|\tilde{u}\|_{\varphi_{x_{0}}}}-\frac{\left\langle\tau_{d} u, \tilde{u}\right\rangle_{\varphi_{x_{0}}} \partial / \partial d\left(\left\|\tau_{d} u\right\|_{\varphi_{x_{0}}}\right)}{\left(\left\|\tau_{d} u\right\|_{\varphi_{x_{0}}}\right)^{2}\|\tilde{u}\|_{\varphi_{x_{0}}}} \\
= & \frac{\left\langle\tau_{d} u^{\prime}, \tilde{u}\right\rangle_{\varphi_{x_{0}}}}{\left\|\tau_{d} u\right\|_{\varphi_{x_{0}}}\|\tilde{u}\|_{\varphi_{x_{0}}}} \\
& -\frac{\left\langle\tau_{d} u, \tilde{u}\right\rangle_{\varphi_{x_{0}}} \partial / \partial d\left(\sqrt{\left\langle\tau_{d} u, \tau_{d} u\right\rangle_{\varphi_{x_{0}}}}\right)}{\left(\left\|\tau_{d} u\right\|_{\varphi_{x_{0}}}\right)^{2}\|\tilde{u}\|_{\varphi_{x_{0}}}} \\
= & \frac{\left\langle\tau_{d} u^{\prime}, \tilde{u}\right\rangle_{\varphi_{x_{0}}}}{\left\|\tau_{d} u\right\|_{\varphi_{x_{0}}}\|\tilde{u}\|_{\varphi_{x_{0}}}} \\
& -\frac{\left\langle\tau_{d} u, \tilde{u}\right\rangle_{\varphi_{x_{0}}}\left\langle\tau_{d} u, \tau_{d} u\right\rangle_{\varphi_{x_{0}}}^{-\frac{1}{2}}\left\langle\tau_{d} u^{\prime}, \tau_{d} u\right\rangle}{\left(\left\|\tau_{d} u\right\|_{\varphi_{x_{0}}}\right)^{2}\|\tilde{u}\|_{\varphi_{x_{0}}}} \\
= & \frac{\left\langle\tau_{d} u^{\prime}, \tilde{u}\right\rangle_{\varphi_{x_{0}}}}{\left\|\tau_{d} u\right\|_{\varphi_{x_{0}}}\|\tilde{u}\|_{\varphi_{x_{0}}}}-\frac{\left\langle\tau_{d} u, \tilde{u}\right\rangle_{\varphi_{x_{0}}}\left\langle\tau_{d} u^{\prime}, \tau_{d} u\right\rangle}{\left(\left\|\tau_{d} u\right\|_{\varphi_{x_{0}}}\right)^{3}\|\tilde{u}\|_{\varphi_{x_{0}}}}
\end{aligned}
$$

where $\|\tilde{u}\|$ is the reference template matching coefficient and $\left\|\tau_{d} u\right\|_{\varphi_{x_{0}}}$ represents the joint template matching coefficient of the image. The gradient decomposition method ${ }^{18}$ is adopted for feature decomposition, to realize contour feature extraction and reconstruction of the three-dimensional spiral
CT damage image. The contour reconstruction expression of the image is obtained as follows:

$$
\begin{aligned}
d_{i}^{k+1}= & (1-\omega) d_{i}^{k} \\
& +\frac{\left(\begin{array}{c}
\sum_{j \in N-(i)}\left(\Theta_{i} \frac{g_{i}+g_{j}}{2}+\left(1-\Theta_{i}\right) \frac{\Psi_{i}^{\prime}+\Psi_{j}^{\prime}}{2}\right) d_{j}^{k+1} \\
+\sum_{j \in N^{+}(i)}\left(\Theta_{i} \frac{g_{i}+g_{j}}{2}+\left(1-\Theta_{i}\right) \frac{\Psi_{i}^{\prime}+\Psi_{j}^{\prime}}{2}\right) d_{j}^{k}-\frac{D_{i}}{\alpha}
\end{array}\right)}{\sum_{j \in N(i)}\left(\Theta_{i} \frac{g_{i}+g_{j}}{2}+\left(1-\Theta_{i}\right) \frac{\Psi_{i}^{\prime}+\Psi_{j}^{\prime}}{2}\right)} .
\end{aligned}
$$

The above formula represents the gray pixel feature of the three-dimensional spiral CT damage image. Kalman filtering ${ }^{19}$ is done to the image by the information fusion method, ${ }^{20}$ thus realizing contour reconstruction of the three-dimensional image. The expression of the contour feature is obtained as follows:

$$
\begin{gathered}
\dot{x}=V \cos \theta \cos \psi_{V}, \quad \dot{y}=V \sin \theta, \\
\dot{z}=-V \cos \theta \sin \psi_{V}, \\
\dot{\vartheta}=\omega_{y} \sin \gamma+\omega_{z} \cos \gamma, \\
\dot{\psi}=\frac{\left(\omega_{y} \cos \gamma-\omega_{z} \sin \gamma\right)}{\cos \vartheta} .
\end{gathered}
$$

In the above system of equations, $x, y$ and $z$ are the edge pixel set. Based on the above analysis, feature extraction and reconstruction of the threedimensional spiral CT damage image are carried out, and adaptive feature matching capability of the image and localization ability of abnormal feature points are improved.

\subsection{Contour reconstruction and design of three-dimensional spiral $C T$ damage image}

In order to realize contour reconstruction of threedimensional spiral CT damage image, first, a model for image feature analysis is established, and the gird model matching method is adopted for image texture rending, and then a uniformly distributed vertex model of three-dimensional spiral CT damage image is constructed. The gird distribution model of spiral CT image has strong anti-interference ability and can improve the image recognition rate. A gird distribution model of spiral CT image is obtained as shown in Fig. 2.

Texture rendering and feature decomposition are carried out to CT image in the gird model shown in 


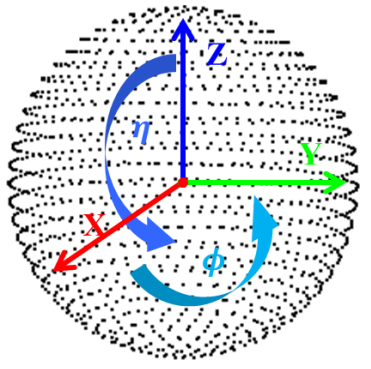

(a) Uniformly distribution gird

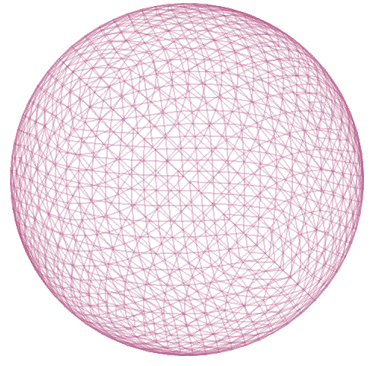

(b) Texture information rendering
Fig. 2. Gird distribution model of three-dimensional spiral CT image.

Fig. 2. The distribution matrix of pixel sequence of three-dimensional spiral CT damage image is set and described as:

$$
D=\left[\begin{array}{cc}
I_{x}^{2} & I_{x} I_{y} \\
I_{x} I_{y} & I_{y}^{2}
\end{array}\right]
$$

In the formula, $I_{x}$ represents the pixels of the three-dimensional spiral CT damage image in the $x$-direction, and $I_{y}$ represents the pixels of the threedimensional spiral CT damage image in the y-direction. By using the cost constraint analysis method, the sampling point density of threedimensional spiral CT damage image is obtained as follows:

$$
E\left(\mathbf{T}_{n}\right)=\frac{1}{N} \sum_{i=0}^{N-1}\left\|p_{i}-\mathbf{T}_{n}\left(g_{i}^{\prime}\right)\right\|^{2}+\tau \cdot \Phi\left(\mathbf{T}_{n}\right) .
$$

With the adaptive weighted control method, the inverse weight $f\left(g_{i}\right)$ of the CT image is obtained as:

$$
f\left(g_{i}\right)=c_{1} \tilde{\lambda}_{i} \frac{\sum_{j=0}^{N_{n p}} \rho_{j} \boldsymbol{v}_{i j} /\left|\boldsymbol{v}_{i j}\right|^{\sigma_{1}}+\varepsilon}{\sum_{j=0}^{N_{n p}} \rho_{j} /\left|\boldsymbol{v}_{i j}\right|^{\sigma_{1}}+\varepsilon} .
$$

In the smooth region of Taubin, interpolation transformation is done to CT image, and the model vertex matching method is used to perform the feature decomposition of edge contour, and then the expression of the edge contour feature decomposition of spiral CT damage image is obtained as follows:

$$
\begin{aligned}
& f\left(\mathbf{G}_{n}\right)=a_{1}+a_{2} x+a_{3} y+a_{4} z+\sum_{i=0}^{n} \gamma_{i} U\left(g_{i}^{\prime}, p_{i}\right) \\
& g\left(\mathbf{G}_{n}\right)=b_{1}+b_{2} x+b_{3} y+b_{4} z+\sum_{i=0}^{n} \theta_{i} U\left(g_{i}^{\prime}, p_{i}\right), \\
& h\left(\mathbf{G}_{n}\right)=c_{1}+c_{2} x+c_{3} y+c_{4} z+\sum_{i=0}^{n} \omega_{i} U\left(g_{i}^{\prime}, p_{i}\right)
\end{aligned}
$$

where $\Phi\left(\mathbf{T}_{n}\right)$ is given by the following formula:

$$
\Phi\left(\mathbf{T}_{n}\right)=\boldsymbol{\gamma}^{T} \mathbf{H} \boldsymbol{\gamma}+\theta^{T} \mathbf{H} \theta+\boldsymbol{\omega}^{T} \mathbf{H} \boldsymbol{\omega} .
$$

Based on the construction of the above model, the contours of the three-dimensional spiral CT image are reconstructed, and the pixel reconstruction of edge contour region is performed in the gray pixel region.

The flowchart of the algorithm for reconstruction is shown in Fig. 3.

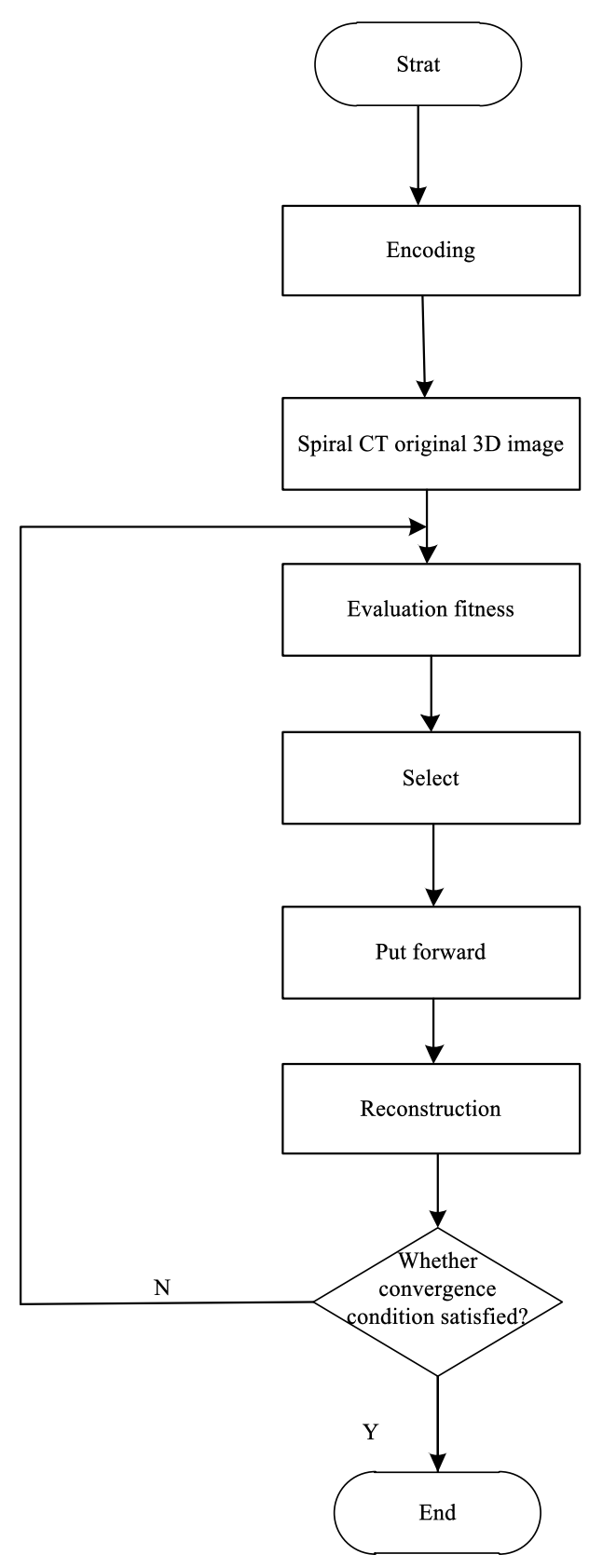

Fig. 3. Flowchart of the algorithm for three-dimensional spiral CT damage image. 
Table 1. Performance comparison of contour reconstruction of three-dimensional spiral CT damage image.

\begin{tabular}{lcccccc}
\hline Method & $\begin{array}{c}\text { Number of } \\
\text { prior points }\end{array}$ & $\begin{array}{c}\text { Signal-to-noise ratio } \\
\text { of peak output (dB) }\end{array}$ & $\begin{array}{c}\text { Total number of } \\
\text { sampling sites }\end{array}$ & $\begin{array}{c}\text { Time of contour } \\
\text { reconstruction } \\
(\mathrm{ms})\end{array}$ & $\begin{array}{c}\text { Smoothing } \\
\text { time }(\mathrm{ms})\end{array}$ & $\begin{array}{c}\text { Normalization } \\
\text { reconstruction } \\
\text { error }\end{array}$ \\
\hline $\begin{array}{l}\text { Method proposed } \\
\text { in this paper }\end{array}$ & 430 & 66 & 954 & 2.34 & 54 & 0.12 \\
\begin{tabular}{l} 
Traditional method \\
\hline
\end{tabular} & 430 & 68 & 954 & 12.23 & 89 & 0.43 \\
\hline
\end{tabular}

\section{Experimental Analysis}

In order to verify the performance of the proposed method in 3D spiral CT damage image reconstruction, a simulation experiment was carried out. The hardware Envi simulation environment is: Intel ( $\mathrm{R})$ $2.3 \mathrm{GHz}, \mathrm{CPU} 2 \mathrm{~GB}$ memory. It is designed by Matlab simulation tool, and the image reconstruction simulation software platform is VisualStudio 2.010 , the pixels of the collected spiral CT images are 6 million, the resolution is $200 * 200$ and the median error of the edge contour reconstruction is median $=0.45 \mathrm{~mm}$. Based on the above simulation environment and parameter settings, two original 3D CT damage images were selected for contour reconstruction. The original three-dimensional CT damage image is shown in Fig. 4.

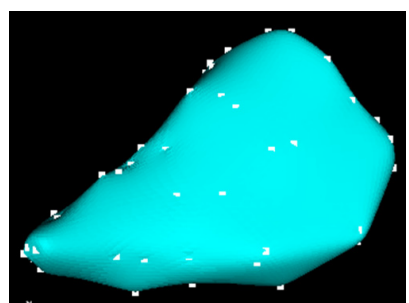

(a)

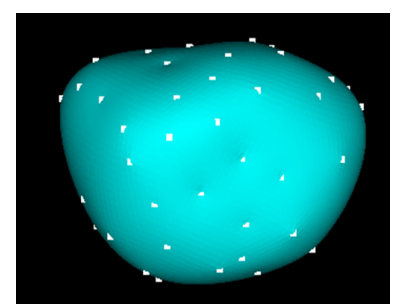

(b)
Fig. 4. Original three-dimensional spiral CT damage image.

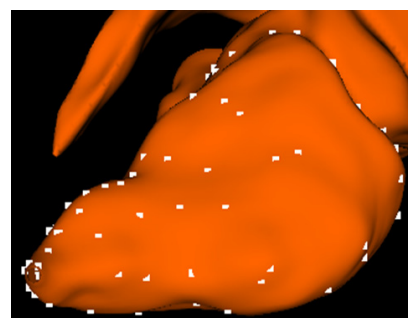

(a)

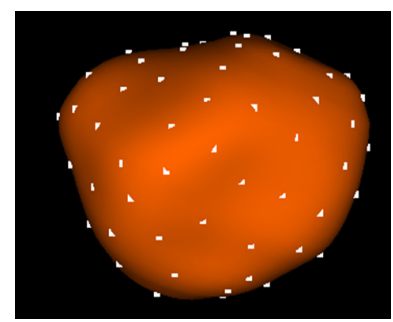

(b)
Fig. 5. Information enhancement of three-dimensional spiral CT damage image.
The image shown in Fig. 4 is taken as research object for three-dimensional contour reconstruction, and the sharpening template enhancement method is adopted for information enhancement processing. Then the result of information enhancement is obtained as shown in Fig. 5.

In Fig. 5, we can see that the spiral CT damage image is enhanced with this method, and the image is clearer and the outline of the feature points is clear, and it is basically consistent with the original image. It shows that the method proposed in this paper can provide the feature points with large information recognition ability and improve the ability of CT image information expression, the adaptive feature matching ability of the image and the location ability of the abnormal feature points. Based on this, the contour reconstruction of the seven different angles of the two original images is

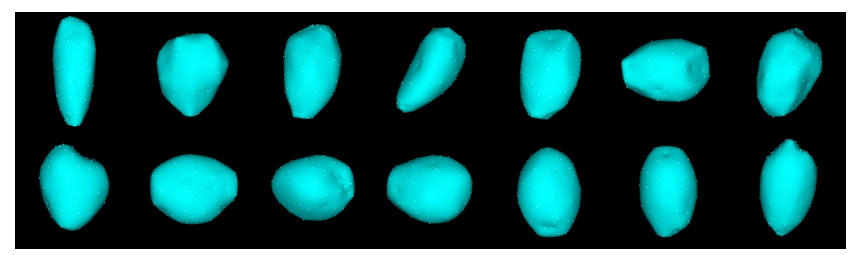

Fig. 6. Contour reconstruction result of three-dimensional spiral CT damage image.

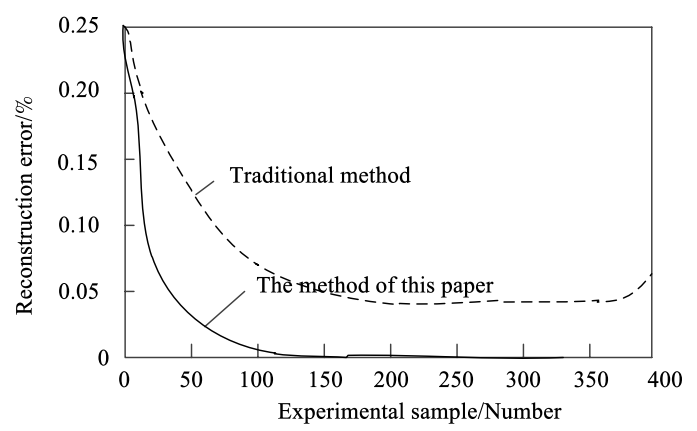

Fig. 7. Comparison of reconstruction error of different methods. 
carried out. Three dimensional spiral CT damage image reconstruction results are shown in Fig. 6.

From Fig. 6, we can see that this method is used to reconstruct seven different angles of two spiral CT damage images. Table 1 shows that the reconstructed results are basically consistent with the original image, and the contour is clear and smooth. Figure 7 shows the reconstruction precision of this method is high.

\section{Conclusion}

(1) The pathological analysis based on spiral CT imaging is based on reconstruction of three-dimensional spiral CT image. Adaptive reconstruction of spiral CT image is reconstructed through information feature extraction and reconstruction technologies, thus realizing highresolution CT imaging and radiography. In this paper, a contour reconstruction method of threedimensional spiral CT image based on sharpening template enhancement technique is proposed.

(2) Noise separation is done to three-dimensional spiral CT damage image. The active contour Lasso model is adopted to extract contour features of three-dimensional spiral CT damage image, and the sharpening template enhancement method is adopted for information enhancement processing of spiral CT image. A statistics shape model of three-dimensional spiral CT damage image is established, and the gradient algorithm is adopted for feature decomposition, so as to realize feature extraction and reconstruction of three-dimensional spiral CT damage image, to improve adaptive feature matching ability and abnormal feature point location ability of image.

(3) The results show that the method proposed in this paper can improve the contour reconstruction ability of three-dimensional spiral CT damage image with less overhead and low error.

(4) This method can be used to reconstruct the image contour and can reconstruct the 3D model of the object or scene with very strong sense of reality, enhance the expression of image information and obtain more image information. This method involves many hot areas, such as computer image processing, computer graphics, computer vision and computer-aided design. This method has become a very potential hot field and has very important applications in many fields, such as electronic commerce, space flight, remote sensing mapping, virtual museum and other high-tech fields.

In the future, the research should be focussed on contour reconstruction capability of threedimensional multi-slice spiral CT damage image, image segmentation and reconstruction of 64-bit raw spiral CT scan data.

\section{References}

1. S. K. Meher, "Recursive and noise-exclusive fuzzy switching median filter for impulse noise reduction," Eng. Appl. Artif. Intell. 30, 145-154 (2014).

2. M. H. Hsieh, F. C. Cheng, M. C. Shie et al., "Fast and efficient median filter for removing 1-99\% levels of salt-and-pepper noise in images," Eng. Appl. Artif. Intell. 26(4), 1333-1338 (2013).

3. J. J. Wang, R. D. Wang, W. Li et al., "An information hiding algorithm for HEVC based on intra prediction," J. Optoelectron. Laser 25(8), 1578-1585 (2014).

4. J. Zhang, "Binarization method with local threshold based on image blocks," J. Comp. Appl. 37(3), 827-831 (2017).

5. J. F. Wang, Z. C. Huang, A. M. A. Talab, "New binarization method called $\mathrm{BM}$ aim to optimize detail of image," J. Wuhan Univ. Technol. 36(8), 127-132 (2014).

6. Z. X. Lu, B. B. Zhang, "A new segmentation method of hand dorsal vein image," Microelectron. Comp. 31(8), 25-28 (2014).

7. M. Zhang, W. Chen, L.-W. Chen et al., "Photorefractive long period waveguide grating filter in lithium niobate strip waveguide," Opt. Quant. Electron. 46, 1529-1538 (2014).

8. J. Cao, H.-S. Li, Q. Cai, "Research on feature extraction of image target," Comp. Simul. 30(1), 409-413 (2013).

9. W. Wang, Q. Yan, D. Jin, "Object-oriented remote sensing image classification based on GEPSO model," Comp. Sci. 42(5), 51-53 (2015).

10. H.-C. Luo, Y.-Y. Li, J. Sun, "Filtering method for images based on adaptive neuro-fuzzy interference system," Comp. Sci. 40(7), 302-306 (2013).

11. H. Yu, Z. Liu, Q. Tian, "A spectral prediction model of printer based on RBF neural network," Imaging Sci. Photochem. 33(3), 238-243 (2015).

12. C. Liu, H. B. Zhao, C. S. Li et al., "CSP/SVMbased EEG classification of imagined hand movements," J. Northeast. Univ. (Natu. Sci.), 31(8), 1098-1101 (2010). 
13. F. Grimm, G. Naros, A. Gharabaghi, "Closed-loop task difficulty adaptation during virtual reality reach-tograsp training assisted with an exoskeleton for stroke rehabilitation," Front. Neurosci. 10, 518 (2016).

14. M. Mukaino, T. Ono, K. Shindo et al., "Efficacy of brain-computer interface-driven neuromuscular electrical stimulation for chronic paresis after stroke," J. Rehabil. Med. 46(4), 378-382 (2014).

15. P. Ferrara, T. Bianchi, "Image forgery localization via fine-grained analysis of CFA artifacts," IEEE Trans. Inform. Forens. Security 7(5), 1566-1577 (2012).

16. L. Tengfei, J. Weili, "Automatic line segment registration using Gaussian mixture model and expectation-maximization algorithm," IEEE J. Select. Top. Appl. Earth Observ. Remote Sens. 7(5), 1688-1699 (2014).
17. L. Siwei, P. Xunyu, Z. Xing, "Exposing region splicing forgeries with blind local noise estimation," Int. J. Comp. Vis. 110(2), 202-221 (2014).

18. Y. Huang, Z. Wu, L. Wang et al., "Feature coding in image classification: A comprehensive study," IEEE Trans. Patt. Anal. Mach. Intell. 36(3), 493-506 (2014).

19. L. L. Rui, P. Zhang, H. Q. Huang et al., "Reputationbased incentive mechanisms in crowdsourcing," $J$. Electron. Inform. Technol. 38(7), 1808-1815 (2016).

20. Y. Zhang, C. Jiang, L. Song et al., "Incentive mechanism for mobile crowdsourcing using an optimized tournament model," IEEE J. Select. Areas Commun. 35(4), 880-892 (2017). 